\title{
Application of Satellite Observations to Manage Natural Disasters in the Lake Victoria Basin
}

\author{
Shahid Habib, NASA Goddard Space Flight Center, Greenbelt, Maryland, USA \\ Fritz Policelli, NASA Goddard Space Flight Center, Greenbelt, Maryland, USA \\ Dan Irwin, NASA Marshall Space Flight Center, Huntsville, Alabama, USA \\ Tesfaye Korme, Regional Center for Mapping and Resource Management, Nairobi, Kenya \\ Bob Adler, University of Maryland. College Park, Maryland, USA \\ Yang Hong, University of Oklahoma, Norman, Oklahoma, USA
}

Lake Victoria, the second largest fresh water lake in the Eastern part of Africa is a vital natural resource for the economic well being and prosperity of over 30 million people located in riparian regions of Uganda, Kenya and Tanzania. It covers a large area of about $68,870 \mathrm{~km}^{2}$ and produces a GDP of about US $\$ 30$ billion per year. The region is also very much prone to natural disasters such as severe floods during heavy precipitation periods in the Eastern part of Africa. In addition to floods, the precipitation also produces large infestations of mosquito larvae due to the standing water in many areas. This further causes multiple vector borne diseases such as Malaria, Rift Valley Fever and more. These problems are of serious concern and require active and aggressive surveillance and management to minimize the loss of human and animal lives and property damage. Satellite imagery and observations along with the in situ measurements provide a great tool to analyze and study this area and inform the policy makers to make calculated policy decisions which are more beneficial to the environment. Recently, NASA and USAID have joined forces with the Regional Center for Mapping of Resources for Development (RCMRD) located in Nairobi, Kenya to utilize multiple NASA sensors such as TRMM, SRTM and MODIS to develop flood potential maps for the Lake Victoria Basin. The idea is to generate a flood forecasts and "nowcasts" that can be sent to the disaster management organizations of Uganda, Kenya, and Tanzania. Post flood event satellite imagery is becoming a common tool to assess the areas inundated by flooding. However, this work is unique undertaking by utilizing land imaging and atmospheric satellites to build credible flood potential maps. At same time, we are also studying the potential occurrence and spread of Rift Valley Fever disease based on the short term climate records and precipitation data. These activities require multi-nation coordination and agreements and multiple operational agencies within each respective country. It also requires credible in situ data such as precipitation, river flow rates and lake levels to further validate the global and regional flood models and algorithms. This also requires a considerable amount of training and capacity building for the RCMRD experts who will help us validate the model results and eventually transition it for operational use. In a final analysis, Disaster management and humanitarian aid organizations need accurate and timely information for making decisions regarding deployment of relief teams and emergency supplies during major floods. Flood maps based on the use of satellite data have proven extremely valuable to such organizations for identifying the location, extent, and severity of these events. However, despite extraordinary efforts on the part of remote sensing data providers to rapidly deliver such maps, there is typically a delay of several days or even weeks from the on-set of flooding until such maps are available to the disaster management community. This paper 
summarizes efforts at NASA to address this problem through development of an integrated and automated process of a) flood forecasting b) flood detection, c) satellite data acquisition, d) rapid flood mapping and distribution, and e) validation of flood forecasting and detection products. 